rev.relac.int.estrateg.segur.12(1):75-102,2016

\title{
PRÁCTICAS DE SEGURIDAD EN AMÉRICA DURANTE LA POSGUERRA FRÍA (1992-2010): ¿COMPLEJOS REGIONALES O COMUNIDADES PLURALISTAS DE SEGURIDAD?*
}

\author{
Marco Vinicio Méndez-Coto**
}

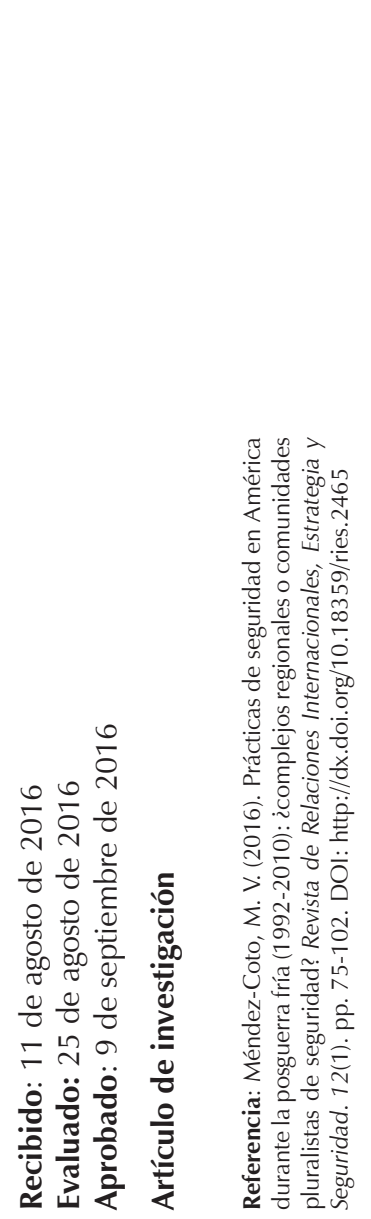

\section{Resumen}

El nuevo regionalismo de seguridad en América, durante la posguerra fría, ha fallado en aportar suficiente evidencia empírica comparada para determinar la presencia de Complejos regionales de seguridad (CRS) o Comunidades pluralistas de seguridad (CPS). Frente a este problema el estudio busca responder ien qué medida las prácticas de seguridad prevalecientes en el continente durante la posguerra fría (1992-2010) permiten diferenciar los CRS o CPS en los niveles regionales y subregionales?

\footnotetext{
Artículo de investigación, resultado parcial de la investigación doctoral "Enfrentando agresiones externas. Estados pequeños y complejos regionales de seguridad: los casos de Costa Rica y Ecuador" de la Facultad Latinoamericana de Ciencias Sociales (FLACSO) - Ecuador. Se agradece especialmente al Departamento de Estudios Internacionales y Comunicación de FLACSO - Ecuador por ser el espacio desde donde surge el presente artículo.

** Candidato a Doctor en Estudios Internacionales por FLACSO Ecuador. Premio de estudios de posgrado de la Organización de Estados Americanos, período 2016-2018. Cuenta con una Maestría en Derechos Humanos y Educación para la Paz y una Licenciatura en Relaciones Internacionales, ambos de la Universidad Nacional de Costa Rica donde se desempeña como profesor investigador de la Escuela de Relaciones Internacionales. Ha publicado libros y más de una decena de artículos en revistas científicas indexadas. Contacto: marco.mendez.coto@gmail.com / mvmendezfl@flacso.edu.ec
} 
Las prácticas de seguridad se estudiaron a partir de dos indicadores: las disputas interestatales militarizadas mediante estadísticas descriptivas y modelos econométricos, y la persistencia de la hipótesis de conflicto en instrumentos programáticos de defensa, mediante el análisis de contenido. Se concluye que el abordaje más apropiado lo ofrecen los CRS dada la persistencia de la amenaza y del uso de la fuerza como práctica de seguridad. La primacía de la agenda estadounidense en América del Norte, América Central y el Caribe implican la presencia de un CRS centrado por su proyección de poder e influencia mundial, mientras que en América del Sur se presenta un CRS estándar. En el nivel subregional, América Central y la zona Andina constituyen subcomplejos, mientras que el Cono Sur una comunidad pluralista de seguridad.

Palabras clave: América; Disputas interestatales militarizadas; Seguridad internacional.

\title{
SECURITY PRACTICES IN AMERICA DURING THE POST-COLD WAR (1992-2010): REGIONAL COMPLEXES OR PLURALISTIC SECURITY COMMUNITIES?
}

\begin{abstract}
The new security regionalism in America during the post-Cold War period has failed to offer enough comparative empirical evidence in order to determine the presence of Regional Security Complexes (RSC) or Pluralistic Security Communities (PSC). In the face of this issue, this study intends to answer, to what extent do security practices prevailing in the continent during the post-Cold War (1992-2010) allow the differentiation between RSC and CPS at the regional and sub-regional level? Security practices were analyzed taking into account two indicators: militarized inter-state disputes by means of descriptive statistics and econometric models, and the persistence of the hypothesis of conflict in defense programmatic instruments through content analysis. The conclusion is that the most appropriate approach is offered by RSC due to the persistence of the threat and the use of force as security practice. The primacy of the American agenda in North America, Central America, and the Caribbean implies the presence of a RSC centered on its power projection and worldwide influence, while in South America there is a standard RSC. At the sub-regional level, Central American and the Andean zone constitute sub-complexes, whereas in the Southern extreme of the continent the occurrence is that of a pluralistic community
\end{abstract}


Keywords: America; militarized inter-state disputes; international security.

\section{PRATICAS DE SEGURANÇA NA AMÉRICA DURANTE A PÓS-GUERRA FRIA (1992-2010): COMPLEXOS REGIONAIS OU COMUNIDADES PLURALISTAS DE SEGURANÇA?}

\section{Resumo}

O novo regionalismo de segurança na América durante a pós-guerra fria falhou em aportar suficiente evidência empírica comparada para determinar a presença de Complexos regionais de segurança (CRS) ou Comunidades pluralistas de segurança (CPS). Em frente a este problema o estudo procura responder em qual medida as práticas de segurança prevalecentes no continente durante a pósguerra fria (1992-2010) permitem diferenciar os CRS o CPS nos níveis regionais e sub-regionais? As práticas de segurança se estudaram a partir dos indicadores: as disputas interestatais militarizadas mediante estadísticas descritivas e modelos econométricos, e a persistência da hipóteses de conflito em instrumentos programáticos de defesa mediante a análises do conteúdo. Se conclui que a abordagem mais apropriada oferecem os CRS dada a persistência da ameaça e do uso da força como prática de segurança. A primicia da agenda estadunidense na América do Norte, América Central e ou Caribe implicam a presença de um CRS centralizado por sua projeção de poder e influência mundial, enquanto que na América do Sul se apresenta um CRS estandardizado. No nível sub-regional América Central e a zona Andina constituem sub-complexos, enquanto que o Cono Sul uma comunidade pluralista de segurança.

Palavras-chave: América; Disputas interestatais militarizadas; Segurança internacional.

\section{Introducción}

Las dinámicas de seguridad de la segunda década del siglo XXI hacen reflexionar sobre las lógicas que subyacen en los procesos del regionalismo contemporáneo. Frasson-Quenoz (2014) ha discutido si lo imperante en América consiste en procesos de institucionalización de Comunidades pluralistas de seguridad (CPS) o bien de Complejos regionales de seguridad (CRS): entre la configuración de identidades comunes, en la cual el conflicto se torna en un contingente más que en una regularidad o más bien prevalecen dinámicas determinadas por el balance 
de poder, la suspicacia y alineamientos en torno de liderazgos regionales ${ }^{1}$.

Esta discusión analítica debe ser provista de mayor evidencia empírica y de estudios comparados, siendo el propósito de este trabajo. A diferencia de los enfoques del nuevo regionalismo que estudia la dimensión discursiva con el objetivo de discernir proyectos políticos particulares o de abordajes que se limitan a la perspectiva geográfica, este artículo se decanta por una perspectiva desde la regionalización, o mejor dicho desde las "prácticas de seguridad" que - a pesar de los discursos relativos a la integración y la cooperación -, subyacen en las dinámicas de la política y la defensa. En este sentido, el dilema que se busca responder es ien qué medida las prácticas de seguridad prevalecientes en el continente durante la posguerra fría (1992-2010) permiten diferenciar los CRS o CPS en los niveles regionales y subregionales?

Las prácticas de seguridad serán entendidas como las dinámicas fácticas que prevalecen en las relaciones interestatales, y para su abordaje se utilizan dos técnicas de investigación. Desde el punto de vista cuantitativo se estudian las disputas interestatales militarizadas como indicadores objetivos respecto de la hostilidad y conflictividad y teorizadas como antesala de las guerras interestatales, para ello se utilizan técnicas econométricas para discernir la probabilidad y la prevalencia del uso o amenaza de la fuerza en los niveles subregionales y regionales. En complementación como estrategia cualitativa se utiliza el análisis de contenido en instrumentos programáticos relativos a la defensa externa (Constituciones políticas, Libros blancos, políticas nacionales o planes) para identificar la persistencia o no de las amenazas tradicionales (agresiones externas) como otro indicador de dichas prácticas.

El artículo se organiza mediante las siguientes secciones: la primera analiza los complejos regionales y las comunidades pluralistas en el marco del nuevo regionalismo de seguridad, argumentándose que para el caso del continente americano la "variable" se da a nivel de las prácticas de seguridad pues la arquitectura institucional, con independencia de la categoría en uso, es una "constante". La segunda sección profundiza en las disputas militarizadas como indicador objetivo de las prácticas de seguridad, realizando en primera instancia una

Ver reseña de Méndez-Coto (2016b) por cuanto Frasson-Quenoz no utiliza la perspectiva de las prácticas sino geográfica, limitándose a estudiar América del Norte y América del Sur sin resaltar las dinámicas de los subcomplejos y omitiendo a América Central y el Caribe. 
diferenciación respecto del conflicto limítrofe y posteriormente dando cuenta del estado de la literatura en la temática. La tercera sección ofrece el diseño metodológico utilizado en el trabajo para dimensionar el alcance de sus resultados, los cuales constituyen una propuesta innovadora para generar evidencia empírica en la discusión relativa al regionalismo de seguridad.

La cuarta sección estudia las dinámicas de hostilidad y conflictividad en el continente, afirmando que la amenaza y el uso de la fuerza han estado presentes en las décadas de 1990 y 2000 en América, y por esta razón la hipótesis de conflicto interestatal no ha podido ser descartada, lo cual se evidencia al estudiar los instrumentos programáticos de defensa. Seguidamente se analiza la evidencia aportada para discernir en qué contextos (regionales o subregionales) resulta más adecuado utilizar las categorías en disputa: los CRS o las CPS, para concluir con una síntesis analítica.

\section{El nuevo regionalismo de seguridad: entre el CRS y las CPS}

Los estudios sobre el regionalismo continúan enfrentando el reto conceptual. Como destaca Fawcett, los términos no son fijos y son objeto de múltiples interpretaciones (2005, p. 23). A pesar de lo anterior, existe consenso en identificar dos olas regionalistas a partir de la Segunda Guerra Mundial (Breslin, Higgott \& Rosamond, 2003; Fawcett, 2005; Gómez-Mera, 2008; Hettne \& Söderbaum, 2003). La primera ola del "viejo regionalismo" basada en los esquemas clásicos de integración que tomaban como imagen la experiencia europea; y la segunda ola, denominada del "nuevo regionalismo" caracterizado por un mundo en transición al multipolarismo, el declive de la hegemonía estadounidense y la intensificación de la globalización y la desterritorialización (Cf. Méndez-Coto 2012, 2014, 2015, 2016a).

A pesar de esa ausencia de consensos, es posible identificar ideas comunes para estudiar el "regionalismo", la " regionalización" y las "regiones" en el nuevo regionalismo. Estos autores insisten en que por 'regionalismo' debe entenderse un proyecto o curso de acción (Fawcett, 2005, p. 24; Hettne, 2004, p. 28) donde las unidades cooperan y coordinan en una región; además, según Hettne y Söderbaum, analíticamente se refiere a una ideología que busca el "impulso de un orden regionalista, ya sea en una zona geográfica determinada o como un tipo de orden mundial" (2003, p. 34). Este carácter normativo se asocia con una intensificación de la cooperación, de allí la posibilidad de su institucionalización (Fawcett, 2005, p. 24).

Este proyecto no debe confundirse con lo fáctico, que ha sido 
denominado "regionalización": los procesos empíricos que "provienen de los mercados, de los flujos de comercio e inversión privadas, y de las decisiones empresariales" (Breslin et al., 2003, pp. 13-14) que a su vez llevan a "patrones de cooperación, integración, complementariedad y convergencia" (Hettne \& Söderbaum, 2003, p. 34) o para el caso de este trabajo por las "prácticas de seguridad". Para Hettne y Söderbaum las 'regiones' son construcciones sociales, que se distinguen por "procesos de regionalización que, en diversos campos y niveles, se intensifican y convergen en la misma zona geográfica, por tanto la cohesión y el carácter distintivo de la región, aumentan" (2003, p. 38).

Por otra parte, en el marco del nuevo regionalismo de seguridad Robert Kelly estudia las teorías contemporáneas señalando que su aporte consiste en la distinción de lo regional como nuevo nivel con una dinámica propia. Caracteriza este momento por la introducción de tres nuevas variables: las regiones son abiertas a la influencia del sistema internacional, la estructura global es cerrada; la densidad geográfica es determinante, haciendo que los problemas de seguridad en el nivel local generen dinámicas regionales distinguibles; y la debilidad de muchos Estados hace más preocupante el disenso doméstico que la guerra interestatal (2007, p. 198).

En este contexto de intersección se enmarca el dilema planteado en este trabajo. El "nuevo regionalismo" ha consistido en una propuesta reflectivista liderada por autores europeos en la cual se privilegia la dimensión ideacional y discursiva. No obstante lo anterior, el nuevo regionalismo de seguridad si bien discute en estos parámetros ideacionales también continúa vinculado con los estudios clásicos de seguridad dando importancia a la dimensión material, por la relevancia de la geopolítica y del uso de la violencia. Esto resulta fundamental pues el abordaje propuesto privilegia lo fáctico (prácticas de seguridad) y no lo deontológico.

En este sentido, se busca discernir y comparar dos teorías de la seguridad que explican las dinámicas del regionalismo contemporáneo mediante evidencia empírica. La teoría de los Complejos regionales de seguridad (CRS) propone lo regional como un cuarto nivel de análisis en los estudios internacionales, y ha buscado distinguirse del neorrealismo y del globalismo por la debilidad de estos enfoques al exagerar el papel de la estructura internacional y subestimar el nivel regional (Buzan \& Wæver, 2003, p. 21). Esto se explica porque en la posguerra fría solamente Estados Unidos tiene capacidad global, lo que lleva a plantear una distinción entre las Superpotencias que operan a escala global, las Potencias grandes que operan en más de una región pero no globalmente, y las Potencias regionales que operan dentro de una sola región. Debido a que solamente las 
Superpotencias operan en la escala global, la teoría de la polaridad pierde fuerza, de allí la pertinencia de los CRS como subestructuras que median las dinámicas globales, cuya formación se deriva de la interacción entre la anarquía, el balance de poder y la cercanía geográfica, pues la adyacencia implica mayor interacción de seguridad generando patrones de clústeres de base regional (Buzan \& Wæver, 2003, p. 45). Los CRS son regiones "vistas con los lentes de la seguridad... que no empiezan o dependen de otras conceptualizaciones de 'regionalidad'" y su existencia no es "en términos de la discursividad sobre la 'construcción de regiones'..., sino de los discursos y prácticas de seguridad de los actores" (Buzan \& Wæver, 2003, pp. 43-44; 48) tomando distancia pero dialogando con la perspectiva reflectivista del "nuevo regionalismo", e indicando que la estructura esencial y el carácter de los CRS se da por dos tipos de relaciones: las relaciones de poder en términos de balances y los patrones de rivalidad como construcciones sociales.

Los CRS son mutuamente excluyentes de tal forma que ofrecen un panorama global y se articulan a partir del tipo de hegemonía prevaleciente. Los CRS "estándar" son aquellos definidos por Potencias regionales y pueden ser uni, bi o multipolares (caso de Brasil en América del Sur) y los CRS "centrados" son aquellos donde una Superpotencia, una Gran potencia o una Institución determina la polaridad, como ocurre con Estados Unidos, Rusia o la Unión Europea. Asimismo, Buzan y Wæver (2003) identifican tres subcomplejos en el continente americano: las subregiones de América Central, la zona andina y el Cono Sur. En relación con las Comunidades pluralistas de seguridad, siguiendo a Frasson-Quenoz, se considera que es aquella en donde "una comunidad política cuyos miembros han adquirido la convicción que 'sus problemas sociales comunes pueden y deben ser resueltos por mecanismos de cambio pacífico, [es decir] por la vía de procedimientos institucionalizados, sin recurrir a la violencia física'", por lo cual la CPS es el ámbito "en el cual los Estados 'dejan de preocuparse por las amenazas militares en proveniencia de otros miembros de la comunidad para privilegiar los problemas de seguridad que giran alrededor de las temáticas económicas, del medio ambiente y del bienestar social'" (2013, p. 25 citando a Adler y Barnett 1998). Sobre el particular, Ovando Santana estima que "se trata de grupos de Estados, para quienes el uso de la fuerza en la resolución de disputas se ha convertido en algo impensable" (2012, p. 204).

A diferencia del CRS las CPS desafían la noción clásica de la seguridad en términos de la continuidad y la adyacencia geográfica, planteando que las mismas pueden ser discontinuas y transcontinentales, no obstante este sentido es una construcción social e histórica propia de las interacciones por lo cual también pueden ser del 
primer tipo (es decir adyacentes). Otra diferencia fundamental consiste en el tipo de cultura anárquica prevaleciente definida por la orientación del Yo frente al Otro en términos del uso de la violencia (Wendt, 1999, pp. 247-249), lo que permite distinguir el sistema de seguridad entre el CRS y la CPS. En el CRS impera una cultura anárquica hobbesiana o lockeana donde el uso de la violencia es un comportamiento aceptado, mientras que en la CPS con una cultura anárquica kantiana, donde no lo es. Por esta razón la concepción de una región como CRS o CPS se vincula con su tipo de paz pues la CPS entendida por la confianza mutua, previsibilidad del cambio pacífico y la renuncia a la resolución de disputas mediante la violencia (en este artículo como diputas militarizadas) se vincula con la "paz positiva", mientras que el CRS se acerca a las visiones más realistas de la "paz negativa" y de la "paz híbrida" como ausencia temporal del conflicto y de violencia directa.

En el continente americano existe una arquitectura de seguridad consolidada a través de los años, amparada en la Organización de Estados Americanos, el Tratado Americano de Soluciones Pacíficas (Pacto de Bogotá) y en menor medida por el Tratado Interamericano de Asistencia Recíproca (Tratado de Río o TIAR), diseñada para el mantenimiento de la seguridad colectiva y el arreglo pacífico de disputas mediante consultas diplomáticas (Herz, 2008, p. 11). A pesar de lo anterior, Mares señala la ausencia de una norma sobre el "no primer uso de la fuerza militar" pues la arquitectura "está diseñada para activarse después de que un gobierno ha decidido que la militarización es una buena idea" (2014, p. 430). Esta arquitectura de seguridad reflejada en diversas normas constituye una "constante" si se busca comparar las dinámicas regionales y subregionales dentro del continente, pues los Estados americanos se encuentran socializados en ellas y forman parte de la dinámica histórica y política en la cual desarrollan sus interacciones. Por ello, se estima innovador realizar un abordaje no desde el diseño institucional o de las aspiraciones políticas sino desde las prácticas de seguridad de la posguerra fría (1992 hasta 2010), pues la ausencia o la presencia de la amenazas o del uso de la fuerza armada consiste en un indicador objetivo para determinar si, allende las normas prevalecientes, el comportamiento de los Estados se vincula con las dinámicas de los CRS o de las CPS, de allí que las disputas interestatales militarizadas consisten en un sendero de investigación útil para la discusión académica del nuevo regionalismo de seguridad.

\section{Las disputas interestatales militarizadas en América}

Para estudiar las disputas interestatales militarizadas en el continente, el primer paso consiste en realizar una diferencia conceptual respecto de los "conflictos limítrofes". Para Hensel y Mares éste es aquel donde existe "desacuerdo 
sobre dónde se deberán trazar los límites físicos y jurisdiccionales entre vecinos... Esos límites fronterizos podrían ser en tierra, agua o aire" (1998, p. 5; 2003, p. 50); mientras que la "disputa interestatal militarizada" es definida como los "casos históricos unificados en los que la amenaza, exhibición o uso de la fuerza militar - por debajo de la guerra - por un Estado, se dirigen explícitamente hacia el gobierno, representantes oficiales, fuerzas oficiales, propiedad o territorio de otro Estado" (Jones, Bremer \& Singer, 1996, p. 168).

$\mathrm{Si}$ bien es cierto que "conflicto limítrofe" y "disputa interestatal militarizada" suelen utilizarse como sinónimos y categorías intercambiables, lo anterior es erróneo en la medida en que son procesos en niveles analíticos diferenciados ${ }^{2}$. Como señalan Jones, Bremer y Singer (1996, pp. 167168), no todo conflicto fronterizo implica episodios de amenaza o uso de la fuerza; en un mismo conflicto es posible la ocurrencia en el tiempo de más de un evento de amenaza o uso de la fuerza; o bien, las disputas militarizadas no suceden únicamente entre Estados fronterizos, siendo posible el despliegue, uso o amenaza de la fuerza más allá de las fronteras nacionales e incluso continentales.
La diferenciación entre conflictos limítrofes y disputas militarizadas tiene consecuencias empíricas respecto de su frecuencia e intensidad, y los estudios más rigurosos se han hecho utilizando las bases de datos "Militarized Interstate Disputes" del proyecto "Correlates Of War. En este sentido, Mares encontró que en América solamente el $42 \%$ de los conflictos limítrofes se correlacionaban con las disputas militarizadas entre 1990 y 2001, por lo que "hay mayores probabilidades de que no entren en esa categoría [de disputa militarizada]" (2003, p. 66), fundamentando la pertinencia de estudiar las disputas militarizadas como dinámicas coyunturales que pueden socavar los avances en materia de integración en seguridad y defensa, en la medida que no dependen necesariamente de la existencia previa de conflictos limítrofes, e implican la amenaza o uso de la fuerza interestatal.

Las disputas militarizadas han sido consideradas como antesala para las guerras interestatales, de allí su relevancia en términos de la seguridad internacional siendo un criterio de diferenciación la cantidad de fatalidades anuales: la guerra interestatal es aquella que alcanza o supera la cifra de 1.000 fatalidades

Para Ramsbotham, Woodhouse y Miall es posible diferenciar 'disputa' de 'conflicto' en la medida en que el primero se caracteriza porque requiere arreglo, mientras que el "término conflicto se reserva para luchas más profundas sobre las necesidades humanas insatisfechas que requieren solución” (2011, p. 9). 
anuales, mientras que las disputas militarizadas se ubican en el rango entre 0 y 999 fatalidades anuales (2011, p. 19). Si bien se han llevado a cabo un número importante de investigaciones utilizando las disputas interestatales como indicadores de conflictividad, en estudios internacionales las explicaciones no han sido sencillas pues en algunos casos se constatan postulados e hipótesis derivadas de las corrientes dominantes mientras que en otros casos los mismos postulados son refutados (Martín, 2006, p. 177). Por ello Mares indica que

El registro histórico no admite
explicaciones simples. Las
democracias se han amenazado
e incluso luchado entre sí...
El aumento de la integración
económica no ha detenido a
los Estados de amenazarse y
luchar entre sí... La disuasión ha
fallado y ha tenido éxito... Las
grandes potencias han detenido
los combates..., han mediado en
crisis... y se han quedado a un lado...
las instituciones internacionales han
servido de fachada para los intereses
de la gran potencia de la región...
Estas mismas instituciones han
sido irrelevantes..., y también han
servido de foro para la mediación...
(2001, p. xii).

Ello debe considerarse para evitar aproximaciones deterministas, reconocer la diversidad y el potencial que ofrece el continente respecto del conflicto. En términos analíticos es posible caracterizar el estado de la literatura sobre disputas militarizadas mediante la siguiente lógica: a) los estudios que correlacionan las disputas militarizadas con la democracia (teoría de la paz democrática), b) los estudios que correlacionan las disputas militarizadas con la interdependencia económica (liberalismos), c) los estudios que caracterizan las disputas militarizadas a partir de las lógicas de poder (realismos), d) los estudios que correlacionan las disputas militarizadas con las instituciones internacionales (institucionalismos) y e) estudios críticos que analizan la construcción de discursos e identidades en torno de disputas militarizadas (constructivismos, teorías críticas y posmodernas). Debe notarse la ausencia de estudios para comprender las dinámicas propiamente regionalistas.

América es un continente que ha generado ejemplos concretos que cuestionan la tesis de la paz democrática, específicamente mediante el conflicto bélico sostenido entre Ecuador y Perú en 1995 (Bonilla 1999). Por otra parte, al analizar las disputas militarizadas por díadas y su correlación con el estatus democrático de las partes involucradas, Domínguez afirma que pareciese que "la política democrática fomenta esos conflictos limítrofes y territoriales militarizados" (1998, pp. 31-34; Martín, 2006, p. 179) al estudiar cómo en las dinámicas domésticas se utiliza el conflicto externo como movilizador de adeptos, 
o para impedir acuerdos ${ }^{3}$. No obstante, también señala que en otros casos ha funcionado como garantía para la legitimidad de los tratados (Chile - Argentina), por lo que no existe una respuesta definitiva respecto del historial americano (Cf. modelos econométricos de este estudio).

En relación con la interdependencia económica, la persistente rivalidad entre Colombia y Venezuela en la política contemporánea, es ejemplar. Autores como Mares afirman que "la existencia de una disputa fronteriza como tal no es un impedimento para... la institucionalización de una relación de cooperación económica ni para el crecimiento de esa relación" (2003, p. 71). Sobre la misma línea Martín asevera que en América del Sur la "paz interestatal no fue provocada por volúmenes altos o significativos de comercio bilateral e interdependencia económica" (2006, p. 179). Contrario sensu, en el nivel internacional Li y Sacko explican por qué el conflicto sí afecta al comercio, encontrando una respuesta en las expectativas de riesgo de las firmas (2002, pp. 3334), y Simmons plantea que "el mero hecho de disputar una frontera tiene un impacto negativo significativo en el comercio bilateral de países contiguos" (2006, p. 252).
Al estudiar las dinámicas de poder -capacidades materiales — las hipótesis realistas también han sido puestas en cuestionamiento. En el nivel internacional T.V. Paul analizaba el hecho de que Estados más débiles, en comparación con su adversario, iniciaran guerras (1994, pp. 14-17). Otros trabajos han cuestionado el efecto del balance ofensivo-defensivo en la incidencia del conflicto violento en el nivel sistémico, planteando la necesidad de conocer las motivaciones e intereses estatales en las díadas (Gortzak, Haftel \& Sweeney, 2005, p. 86). En el caso sudamericano Martín plantea que, a pesar de encontrarse las principales condiciones realistas, la ausencia de una gran guerra intrarregional representa una "anomalía" (2006, pp. 177-178). Por su parte, Mares y Bernstein analizan el papel de la hegemonía estadounidense entre 1884-1993 afirmando que "por sí misma la hegemonía es insuficiente para explicar el uso de la fuerza en las políticas exteriores de América del Sur", y que tampoco "significó la paz absoluta en Centroamérica o en el Caribe", aunque en otras ocasiones sí intervino mediante sanciones (1998, pp. 57-59; Pion-Berlin \& Trinkunas, 2007, p. 82).

Finalmente una serie de estudios constructivistas, críticos y posmodernos señalan que estos enfoques

Sobre el particular Gibler y Tir (2010) analizan la relación entre la transferencia pacífica de territorio (establecimiento negociado de fronteras) con la desmilitarización, la estabilidad fronteriza y transición democrática, encontrando un coeficiente positivo. Por el contrario, plantean que el régimen democrático no incide en tales transferencias pacíficas de territorio. 
cuantitativos-racionalistas presentan carencias, como: a) "la escasa relevancia de los grupos de seres humanos reales y concretos"; b) "la dificultad de profundizar en las causas de un conflicto concreto desde un análisis orientado a la predicción [o la prospectiva estratégica]", y c) "la ausencia de cualquier tipo de elemento simbólico o identitario en la conceptualización de las fronteras en la mayor parte de estos trabajos" (Cairo \& Lois, 2014, p. 50). En esta línea argumental, Ayoob cuestiona el funcionamiento de las teorías dominantes en los Estados periféricos afirmando que desconocen la realidad de la mayor parte de la comunidad internacional, siendo necesario adaptar sus postulados e identificar las lógicas de poder ocultas, pues la proliferación de conflictos interestatales en el tercer mundo "está íntimamente conectada con el hecho de que el proceso de construcción del Estado-nación usualmente se encuentra inacabado entre países contiguos o cercanos" (2005, p. 22).

\section{Consideraciones metodológicas del estudio}

Cuando se analizan las disputas militarizadas la literatura académica ha trabajado predominantemente sobre la base de disputas por díadas, no obstante la codificación pública está disponible hasta el año 2001. En razón de lo anterior, este artículo innova al realizar una aproximación empírica a partir de la bases de disputas por sucesos únicos y la base de disputas anuales por país permitiendo un período más amplio (1992 y 2010) con observaciones para todos los Estados americanos, y la caracterización de los niveles de hostilidad en el continente durante la posguerra fría.

La base de datos por sucesos únicos ha permitido distinguir las disputas militarizadas por aquellas ocurridas exclusivamente en el continente americano, de tal forma que permite identificar los principales focos geográficosdehostilidadyconflictividad, reconocer la participación de actores extrarregionales, y controlar la proyección de la amenaza y el uso de la fuerza por Estados americanos en el nivel extra continental (por Estados Unidos). De esta manera, la hostilidad, conflictividad y máximas acciones en disputa se caracterizarán mediante estadísticas descriptivas.

La base de datos de disputas anuales por país permite la construcción de modelos econométricos para analizar la correlación y la probabilidad entre las disputas militarizadas y variables independientes provenientes de la literatura académica previamente revisada. El Modelo I consiste de una regresión múltiple robusta donde se utilizan las disputas militarizadas anuales por país (incluyendo las extra continentales), y el Modelo II consiste en una regresión Probit robusta, para la cual se ha creado una variable dicotómica con el valor de "1" si se presentan una o más disputas 
militarizadas anual por país, y " 0 " cuando no se presentan. Los modelos incluyen observaciones anuales para cada uno de los treinta y cinco Estados americanos en el período de 1992-2010 que se ha denominado como "posguerra fría", lo que permite comprender las prácticas de seguridad en el continente. Debe mencionarse que los modelos propuestos no reflejan causalidad sino correlación.

Las variables independientes tratadas en los Modelos I y II son:

a. Subregiones: variable politómica para América Central, Zona Andina, Cono Sur, América del Norte y el Caribe;

b. Gasto militar como porcentaje del Producto Interno Bruto: medición obtenida del "Stockholm International Peace Research Institute" que permite incorporar los aportes realistas;

c. Índice de Libertad: variables tricotómicas construidas a partir del "Freedom in the World Country Ratings". Si bien este Índice no mide la calidad de la democracia, sí mide los derechos y las libertades individuales siendo una aproximación útil a la democracia liberal (IDEA Internacional, 2014, p. 114). Se estableció el valor de "1" para los Estados libres, "2" parcialmente libres y " 3 " cuando no son libres, permitiendo incluir los aportes liberales; y

d. Cantidad de límites estatales: variables tricotómicas construidas a partir del banco de datos "Direct Contiguity Data" (v. 3.1) del proyecto "Correlates Of War", en el cual se incluyen las fronteras terrestres y marítimas pero además aquellos Estados separados por menos de 400 millas náuticas. Siguiendo el trabajo de Collier (1999) se estableció que un Estado americano posee "pocas fronteras" cuando consisten entre 0 y 2; una cantidad "regular de fronteras" entre 3 y 6, y "muchas fronteras" cuando son 7 o más. Esta variable permite recuperar el factor geográfico relevante en el nuevo regionalismo de seguridad.

Por otra parte, se desarrolló una estrategia cualitativa de análisis de contenido en la cual se indagó en todas las constituciones políticas de los Estados americanos, así como en los Libros blancos o en su ausencia en las Políticas o Planes Nacionales de la Defensa. El propósito ha sido identificar la persistencia de las amenazas tradicionales (agresiones externas) en el imaginario político para dilucidar la interiorización de las prácticas de seguridad complementando la amenaza y el uso de la fuerza (disputas militarizadas).

\section{Hostilidad, conflictividad y disputas militarizadas en la Posguerra Fría (1992-2010)}

Esta sección aporta una caracterización de la dinámica de hostilidad y conflictividad en el continente 
durante la posguerra fría (19922010) a partir del banco de datos de disputas militarizadas por sucesos únicos, primeramente comparando el continente con el sistema internacional, seguidamente en términos comparativos entre regiones (equivalen a los Complejos regionales de seguridad) y finalmente entre las subregiones.

Lo primero que puede decirse es que en el período estudiado ocurrieron 575 disputas interestatales militarizadas en el nivel mundial, de las cuales 56 sucedieron en el continente americano representando el 9,7\%. De esas disputas militarizadas, geográficamente ocurrieron: 5 en América del Norte, 19 en América Central, 6 en el Caribe, 23 en la zona Andina y 3 en el Cono Sur. Al analizar la frecuencia anual de las disputas militarizadas se encuentra que en el nivel mundial han oscilado entre 19 (año 2010) y 39 (año 2005). En el caso americano, como refleja la Figura 1, han oscilado entre 1 (años 2002, 2004 y 2007) y 8 (años 1995, 1997 y 2000), permitiendo dos afirmaciones: durante la posguerra fría las disputas militarizadas siempre estuvieron presentes anualmente en América; no obstante, hay una clara diferencia entre la década de 1990 que acumuló 37, en comparación con la década del 2000, con 19. Por ello, la década del 2000 es significativamente menos conflictiva.

Establecida la evidencia respecto de la militarización de las disputas como desafío a la paz y estabilidad en el hemisferio, a continuación se expondrá si la pertenencia a una región (Complejo regional de seguridad) en específico conlleva alguna diferencia en términos de la hostilidad y la conflictividad en la posguerra fría.

Figura 1: Frecuencia de disputas militarizadas a nivel mundial y americano. 1992-2010

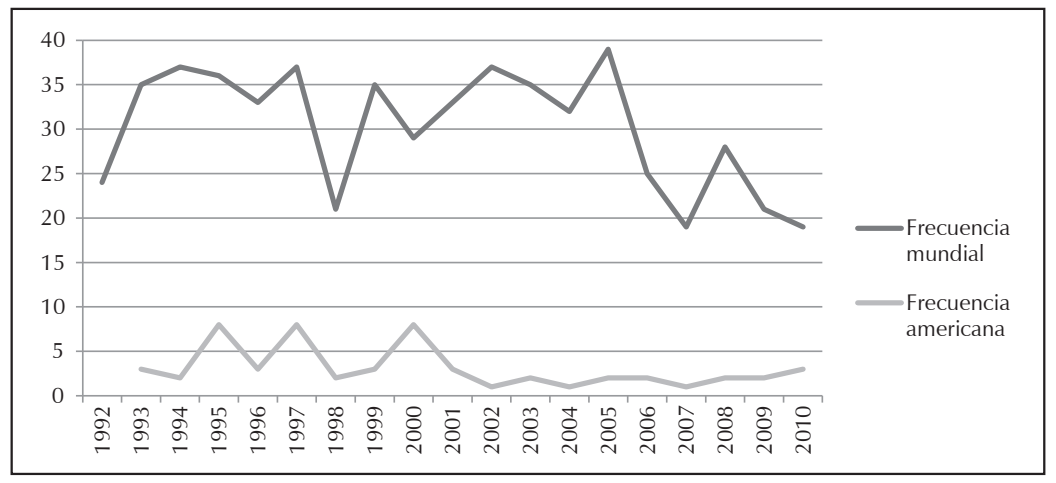

Fuente: Elaboración propia a partir de base de datos Correlates Of War. 
Como se destaca de la Tabla 1, los niveles de hostilidad han sido clasificados en una escala de 1 a 5 , siendo 1 la ausencia de una acción militarizada y 5 la guerra. En el continente americano la totalidad de disputas se ubican entre 2 "amenaza de uso de la fuerza", 3 "despliegue de uso de la fuerza" y 4 "uso de la fuerza". Al comparar los niveles de hostilidad alcanzado en cada CRS, se encuentra en ambos casos al despliegue y uso de la fuerza concentrando prácticamente la totalidad de las disputas, siendo el caso CRS Norte muy levemente más hostil en comparación con el CRS Sur.

Para discernir con mayor certeza las acciones que implican los niveles de hostilidad mencionados se incluye la Tabla 2, en la que se sintetiza las máximas acciones en las 56

Tabla 1: Nivel de hostilidad alcanzado en CRS en América, 1992-2010

\begin{tabular}{|c|c|c|c|c|c|}
\hline \multirow{2}{*}{\multicolumn{2}{|c|}{ Niveles de hostilidad }} & \multicolumn{2}{c|}{ CRS Norte } & \multicolumn{2}{c|}{ CRS Sur } \\
\cline { 3 - 6 } & Frecuencia & Porcentaje & Frecuencia & Porcentaje \\
\hline$[1]$ & Acción no militarizada & 0 & 0 & 0 & 0 \\
\hline$[2]$ & Amenaza de uso de la fuerza & 1 & 3,3 & 0 & 0 \\
\hline$[3]$ & Despliegue de uso de la fuerza & 11 & 36,7 & 11 & 42,3 \\
\hline$[4]$ & Uso de la fuerza & 18 & 60 & 15 & 57,7 \\
\hline$[5]$ & Guerra & 0 & 0 & 0 & 0 \\
\hline \multicolumn{2}{|c|}{ Total } & 30 & 100 & 26 & 100 \\
\hline
\end{tabular}

[\#] Nivel de hostilidad de la disputa.

Fuente: Elaboración propia a partir de base de datos Correlates Of War.

Tabla 2: Máxima acción en disputa en CRS en América, 1992-2010

\begin{tabular}{|c|c|c|c|c|c|}
\hline \multirow{2}{*}{\multicolumn{2}{|c|}{$\begin{array}{c}\text { Acción en disputa en niveles de } \\
\text { hostilidad }\end{array}$}} & \multicolumn{2}{|c|}{ CRS Norte } & \multicolumn{2}{|c|}{ CRS Sur } \\
\hline & & \multirow{2}{*}{$\begin{array}{c}\text { Frecuencia } \\
1\end{array}$} & \multirow{2}{*}{$\begin{array}{c}\text { Porcentaje } \\
3,3 \\
\end{array}$} & \multirow{2}{*}{$\begin{array}{c}\text { Frecuencia } \\
0\end{array}$} & \multirow{2}{*}{$\begin{array}{c}\text { Porcentaje } \\
0\end{array}$} \\
\hline [2] & Amenaza uso de la fuerza & & & & \\
\hline [3] & Demostración de fuerza & 0 & 0 & 1 & 3,8 \\
\hline [3] & Alerta & 1 & 3,3 & 0 & 0 \\
\hline [3] & Fortificación de fronteras & 6 & 20,0 & 3 & 11,5 \\
\hline [3] & Violación de fronteras & 4 & 13,3 & 8 & 30,8 \\
\hline [4] & Bloqueo & 1 & 3,3 & 0 & 0 \\
\hline [4] & Ocupación de territorio & 1 & 3,3 & 1 & 3,8 \\
\hline [4] & Incautación / toma & 9 & 30 & 3 & 11,5 \\
\hline [4] & Ataque & 5 & 16,7 & 8 & 30,8 \\
\hline [4] & Choque / enfrentamiento & 2 & 6,7 & 2 & 7,7 \\
\hline & Total & 30 & 100 & 26 & 100 \\
\hline
\end{tabular}

Fuente: Elaboración propia a partir de base de datos Correlates Of War. 
disputas militarizadas. En el CRS Norte las máximas acciones han sido la incautación / toma con 30\% y la fortificación de fronteras con $20 \%$. En el CRS Sur las máximas acciones han sido los ataques y la violación de fronteras, ambos con 30,8\%. Lo anterior implica que las máximas acciones en disputa, de la misma forma que los niveles de hostilidad, siguen una distribución relativamente similar en ambos casos. Vista la distribución de la hostilidad y conflictividad por CRS, las Tablas 3 y 4 ofrecen el mismo ejercicio pero en los subcomplejos (subregiones). De la Tabla 3 se puede destacar que tanto el subcomplejo América Central como el de zona Andina reflejan dinámicas similares, pues el despliegue y uso de la fuerza porcentualmente no presenta grandes brechas. Un elemento que se destaca es la casi nula presencia de disputas militarizadas en el Cono Sur, siendo que la dinámica de hostilidad y conflictividad se concentra plenamente en la zona Andina, cuando de América del Sur se trata.
Al indagar en las máximas acciones en disputa (Tabla 4) se encuentra que el Cono Sur no solamente es sumamente estable en términos de la ausencia de conflictividad, siendo que 2 de sus 3 disputas han sido diferencias con potencias extra continentales o extrarregionales. En los casos de América Central y la zona Andina los patrones empiezan a distanciarse, planteándose una zona Andina con niveles más intensos de hostilidad que se reflejan en seis choques en comparación con uno en América Central. La mayoría de estas acciones se relacionan con las pugnas entre Ecuador - Perú, Ecuador - Colombia y Venezuela - Colombia.

Los hallazgos destacados implican que el continente no puede verse como una unidad cuando se trata de las prácticas de seguridad, pues la concentración de la amenaza o el uso de la fuerza armada se dan en zonas claramente definidas. Además, permite problematizar el rol de las

Tabla 3: Nivel de hostilidad alcanzado en subcomplejos CRS en América, 1992-2010

\begin{tabular}{|c|c|c|c|c|c|c|c|}
\hline \multirow{2}{*}{\multicolumn{2}{|c|}{ Niveles de hostilidad }} & \multicolumn{2}{c|}{ América Central } & \multicolumn{2}{c|}{ Zona Andina } & \multicolumn{2}{c|}{ Cono Sur } \\
\cline { 3 - 8 } & Frec. & Porcent. & Frec. & Porcent. & Frec. & Porcent. \\
\hline$[1]$ & Acción no militarizada & 0 & 0,0 & 0 & 0 & 0 & 0 \\
\hline$[2]$ & Amenaza de uso de la fuerza & 1 & 5,4 & 0 & 0 & 0 & 0 \\
\hline$[3]$ & Despliegue de uso de la fuerza & 9 & 47,3 & 12 & 52,2 & 0 & 0 \\
\hline$[4]$ & Uso de la fuerza & 9 & 47,3 & 11 & 47,8 & 3 & 100 \\
\hline$[5]$ & Guerra & 0 & 0,0 & 0 & 0 & 0 & 0 \\
\hline & Total & $\mathbf{1 9}$ & $\mathbf{1 0 0}$ & $\mathbf{2 3}$ & $\mathbf{1 0 0}$ & $\mathbf{3}$ & $\mathbf{1 0 0}$ \\
\hline
\end{tabular}

Fuente: Elaboración propia a partir de base de datos Correlates Of War. 
Tabla 4: Máxima acción en disputa en subcomplejos CRS en América, 1992-2010

\begin{tabular}{|c|c|c|c|c|c|c|c|}
\hline \multicolumn{2}{|c|}{$\begin{array}{c}\text { Acción en disputa en niveles de } \\
\text { hostilidad }\end{array}$} & \multicolumn{2}{c|}{ América Central } & \multicolumn{2}{c|}{ Zona Andina } & \multicolumn{2}{c|}{ Cono Sur } \\
\cline { 2 - 9 } & Frec. & Porcent. & Frec. & Porcent. & Frec. & Porcent. \\
\hline$[2]$ & Amenaza uso de la fuerza & 1 & 5,3 & 0 & 0 & 0 & 0 \\
\hline$[3]$ & Demostración de la fuerza & 0 & 0 & 1 & 4,3 & 0 & 0 \\
\hline$[3]$ & Fortificación de fronteras & 5 & 26,3 & 3 & 13 & 0 & 0 \\
\hline$[3]$ & Violación de fronteras & 4 & 21 & 8 & 34,8 & 0 & 0 \\
\hline$[4]$ & Ocupación de territorio & 1 & 5,3 & 1 & 4,3 & 0 & 0 \\
\hline$[4]$ & Incautación / toma & 5 & 26,3 & 2 & 8,7 & 1 & 33,3 \\
\hline$[4]$ & Ataque & 1 & 5,3 & 6 & 26,2 & 2 & 66,7 \\
\hline$[4]$ & Choque / enfrentamiento & 2 & 10,5 & 2 & 8,7 & 0 & 0 \\
\hline
\end{tabular}

Fuente: Elaboración propia a partir de base de datos Correlates Of War.

hegemonías pues en las zonas de influencia inmediata tanto de Estados Unidos (América del Norte) como de Brasil (Cono Sur), la militarización de las disputas es muy baja, y cuando ocurrieron han sido principalmente frente a actores extra continentales. No obstante, las subregiones de interacción entre los liderazgos regionales son altamente inestables (América Central y zona Andina).

Para contrastar el indicador de las disputas interestatales militarizadas se ha realizado un análisis de contenido en Constituciones políticas, Libros blancos o en su ausencia en Planes o Políticas de defensa, para indagar la persistencia de las hipótesis de conflicto interestatal (agresiones externas) que subyace a las prácticas de seguridad. Como resultado, se ha encontrado que en 14 de 21 Constituciones analizadas (67\%) se establece la agresión externa como una amenaza, si se analiza por subregiones la totalidad de Estados tanto del Cono Sur como la zona Andina la establecen. Si se trata de los Libros blancos de defensa, 8 de los 10 publicados reconocen explícitamente la agresión externa como una amenaza potencial, y en ausencia de dichos instrumentos programáticos en 4 de 8 Políticas o Planes Nacionales de Defensa, tal como evidencia la Tabla 5.

Lo anterior problematiza que en regiones como el Cono Sur, caracterizadas por ser altamente estables en términos de la ausencia de disputas militarizadas, la totalidad de los Estados mantengan como hipótesis la agresión externa en sus normas y lineamientos estratégicos, contrario a lo demostrado por la evidencia. Este comportamiento puede explicarse por 
Tabla 5: La agresión externa en constituciones políticas, libros blancos o políticas de defensa de Estados americanos

\begin{tabular}{|c|c|c|c|c|c|c|c|}
\hline \multirow{2}{*}{ Estado } & \multicolumn{2}{|c|}{ Constitución Política } & \multicolumn{2}{|c|}{ Libro Blanco } & \multicolumn{3}{|c|}{ Plan o Política de Defensa (Sin libro blanco) } \\
\hline & $\begin{array}{l}\text { Agresión } \\
\text { externa }\end{array}$ & Referencia & Año & $\begin{array}{l}\text { Agresión } \\
\text { externa }\end{array}$ & Año & $\begin{array}{l}\text { Agresión } \\
\text { externa }\end{array}$ & Documento \\
\hline \multicolumn{8}{|c|}{ Norteamérica } \\
\hline Canadá & - & - & 2008 & - & NA & NA & NA \\
\hline $\begin{array}{l}\text { Estados } \\
\text { Unidos }\end{array}$ & - & - & 2008 & $\sqrt{ }$ & NA & NA & NA \\
\hline México & $\checkmark$ & $\begin{array}{l}\text { Violencia } \\
\text { exterior }\end{array}$ & 2004 & $\sqrt{ }$ & NA & NA & NA \\
\hline \multicolumn{8}{|c|}{ América Central y Caribe } \\
\hline Costa Rica & - & - & - & - & - & - & - \\
\hline Cuba & $\sqrt{ }$ & Agresión militar & - & - & - & - & - \\
\hline El Salvador & $\sqrt{ }$ & Invasión & - & - & - & - & - \\
\hline Guatemala & - & - & 2015 & $*$ & 2012 & - & Pol. Nacional de Seguridad* \\
\hline Honduras & $\sqrt{ }$ & Agresión exterior & 2005 & $\sqrt{ }$ & NA & NA & NA \\
\hline Nicaragua & - & - & 2004 & $\sqrt{ }$ & NA & NA & NA \\
\hline Panamá & $\sqrt{ }$ & Agresión externa & - & - & - & - & - \\
\hline $\begin{array}{c}\text { Rep. } \\
\text { Dominicana }\end{array}$ & $\sqrt{ }$ & Agresión externa & - & - & 2007 & - & $\begin{array}{c}\text { Directiva de Seg. y Defensa } \\
\text { Nacional* }\end{array}$ \\
\hline \multicolumn{8}{|c|}{ Cono Sur } \\
\hline Argentina & $\sqrt{ }$ & Ataque exterior & 2010 & $\sqrt{ }$ & NA & NA & NA \\
\hline Bolivia & $\sqrt{ }$ & Agresión & - & - & 2010 & $\sqrt{ }$ & $\begin{array}{c}\text { Bases Discusión de Doctrina Seg. } \\
\text { y Defensa }\end{array}$ \\
\hline Brasil & $\sqrt{ }$ & $\begin{array}{l}\text { Agresión } \\
\text { extranjera }\end{array}$ & 2012 & $\sqrt{ }$ & NA & NA & NA \\
\hline Chile & $\checkmark$ & Agresión exterior & 2010 & $\sqrt{ }$ & NA & NA & NA \\
\hline Paraguay & $\checkmark$ & Agresión externa & - & - & 1999 & $\sqrt{ }$ & Pol. de Defensa Nacional \\
\hline \multicolumn{8}{|c|}{ Zona Andina } \\
\hline Colombia & $\sqrt{ }$ & $\begin{array}{l}\text { Agresión } \\
\text { extranjera }\end{array}$ & - & - & 2011 & - & $\begin{array}{c}\text { Pol. Integral Seg. y Def. para la } \\
\text { prosperidad* }\end{array}$ \\
\hline Ecuador & $\sqrt{ }$ & Agresión & - & - & 2011 & - & Agenda Política de la Defensa* \\
\hline Uruguay & $\sqrt{ }$ & Ataque exterior & - & - & 2014 & $\sqrt{ }$ & Pol. de Defensa Nacional \\
\hline Perú & - & - & 2005 & $\sqrt{ }$ & NA & NA & NA \\
\hline Venezuela & - & - & - & - & 2007 & $\sqrt{ }$ & Plan "Sucre". Fort. FFAA 2007-2013 \\
\hline
\end{tabular}

Simbología

$\checkmark \quad$ Sí se encuentra presente.

- $\quad$ No se dispone información, o no se encuentra explícitamente.

* S Sí hace referencia a amenazas "externas" y "tradicionales".

NA No aplica porque se satisface la condición anterior.

Fuente: Elaboración propia a partir de RESDAL (2014), Centro de Estudios Estratégicos de Defensa - UNASUR; Comité de Seguridad Hemisférica - OEA, y ministerios de Defensa. 
la inercia institucional argumentada por los teóricos de los regímenes internacionales, por la "reificación de estructuras de ideas en torno a la seguridad nacional" (Ovando Santana, 2012, p. 212) o bien, por la persistencia de amenazas de carácter extrarregional o extra continental (Estados Unidos o Reino Unido) o de subregiones adyacentes (zona Andina). En lazona Andina, 3 de 5 Constituciones políticas reconocen la agresión externa como una amenaza, en los dos Estados ausente se puede encontrar en su Libro blanco (Perú) o en su Plan de Defensa (Venezuela). Las potenciales amenazas externas son más diversas en esta zona, siendo Estados Unidos la principal amenaza y las desconfianzas mutuas frente a países vecinos (Perú - Ecuador - Colombia - Venezuela) dado el historial de conflictividad que prevalece en la subregión.

En América Central y el Caribe se encontró la amenaza de agresión externa en 5 de 8 Constituciones políticas. En el caso de Costa Rica no se encontró en ninguno de los instrumentos programáticos indagados, Nicaragua la establece en su Libro blanco de defensa y Guatemala no la reconoce en su Libro blanco pero sí en su Política Nacional de Seguridad. El caso guatemalteco es de interés pues en su Libro blanco de 2003 descarta las amenazas tradicionales (interestatales), no obstante en el Libro blanco de 2015 recupera la hipótesis de la agresión externa. Finalmente en América del Norte, debido a su tradición jurídica (al igual que en el Caribe), solamente México la incorpora en su Constitución Política, y si bien es cierto que los Libros blancos de Estados Unidos y México establecen la agresión externa como una amenaza, la principal preocupación son las amenazas no tradicionales, específicamente el terrorismo y los grupos criminales transnacionales.

Para complementar el panorama respecto de la distribución geográfica de las disputas militarizadas en el continente, a continuación se ofrecen los principales hallazgos de dos modelos econométricos construidos con la base de datos de disputas anuales por país. Esta base determina anualmente en cuántas disputas ha participado un Estado sin discernir la región o continente donde sucediera o el par antagónico de cada Estado americano.

El Modelo 1 consiste en una regresión lineal múltiple robusta que asocia las disputas anuales por país corrigiendo por subregiones, el PIB en gasto militar, variables tricotómicas provenientes del Índice de libertad y de variables tricotómicas sobre límites fronterizos, como se expuso en la sección metodológica. Tal como fue argumentado, la subregión del Cono Sur se ha presentado como la más estable y menos conflictiva en términos de disputas militarizadas, razón por la cual se ha tomado como referencia (control) para interpretar los resultados de la variable politómica de subregiones. 
Modelo 1: Regresión múltiple: disputas militarizadas por año corrigiendo por subregiones, PIB en gasto militar, Índice de libertad y límites fronterizos, 19922010

\begin{tabular}{|c|c|}
\hline América del Norte & $1.31^{* * *}$ \\
\hline América Central & $0.22^{* * *}$ \\
\hline Zona Andina & $0.44^{* * *}$ \\
\hline Caribe & - \\
\hline Gasto militar (PIB) & $0.25^{* * *}$ \\
\hline Pocas fronteras & $-0.30^{* *}$ \\
\hline Regular fronteras & - \\
\hline Estados libres & $-0.97^{* * *}$ \\
\hline Parcialmente libres & $-1.05^{* * *}$ \\
\hline$N$ & 413 \\
\hline Prob $>F$ & 0.0000 \\
\hline
\end{tabular}

Niveles de significancia: ${ }^{* * *} \mathrm{p}<0.01,{ }^{* *} \mathrm{p}<0.05$; $y^{*} p<0.10$

Los hallazgos son los siguientes: con $1 \%$ de significancia, pertenecer a la subregión América del Norte, América Central y zona Andina se correlaciona con 1,31; 0,22 y 0,44 disputas militarizadas anuales respectivamente, en comparación con el Cono Sur y corrigiendo por las demás variables. La subregión Caribe no mantiene una asociación significativa. Continuando con el Modelo 1 se encuentra que, con $1 \%$ de significancia, un aumento de $1 \%$ del gasto militar como porcentaje del PIB se asocia con 0,25 disputas militarizadas anuales, y en términos de la cantidad de fronteras, con 5\% de significancia poseer pocas fronteras se asocia con -0,30 disputas militarizadas anuales en comparación con los Estados que poseen muchas fronteras. La variable de cantidad regular de fronteras no mantuvo una asociación significativa. En relación con la variable vinculada a la democracia liberal (Índice de libertad), se encuentra con $1 \%$ de significancia que el Estado libre y el Estado parcialmente libre se asocian con -0,97 y -1,05 disputas militarizadas respectivamente, en comparación con los Estados no libres.

Modelo 2: Regresión Probit: probabilidad de disputas militarizadas por año corrigiendo por subregiones, PIB en gasto militar, Índice de libertad y límites fronterizos, 1992-2010

\begin{tabular}{|c|c|c|}
\hline & x-bar & Porcentajes \\
\hline América del Norte & 13.80 & $55.98^{* * *}$ \\
\hline América Central & 22.76 & $25.78^{* * *}$ \\
\hline Zona Andina & 22.27 & $38.13^{* * *}$ \\
\hline Caribe & 13.55 & - \\
\hline Gasto militar (PIB) & 2.06 & $7.06^{* * *}$ \\
\hline Pocas fronteras & 13.80 & - \\
\hline Regular fronteras & 48.66 & - \\
\hline Estados libres & 56.65 & $-69.83 * * *$ \\
\hline Parcialmente libres & 42.85 & $-54.77^{* *}$ \\
\hline$N$ & \multicolumn{2}{|c|}{413} \\
\hline Prob $>$ Chi ${ }^{2}$ & \multicolumn{2}{|c|}{0.0000} \\
\hline Prob. observada & \multicolumn{2}{|c|}{227682 (en x-bar) } \\
\hline Prob. pred & \multicolumn{2}{|c|}{}
\end{tabular}

Niveles de significancia: ${ }^{* * *} \mathrm{p}<0.01,{ }^{* *} \mathrm{p}<0.05$; $y^{*} p<0.10$.

El Modelo 2, mediante una regresión Probit robusta, analiza la probabilidad de las disputas anuales por país corrigiendo por subregiones, el PIB en gasto militar, variables tricotómicas provenientes del Índice de libertad y de variables tricotómicas de límites fronterizos, siguiendo la estructura del Modelo 1 . Con $1 \%$ de significancia, 
pertenecer a la subregión América del Norte, América Central y zona Andina se asocian con 55,98\%, 25,78\% y $38,13 \%$ de probabilidad de disputas anuales por país respectivamente, en comparación con el Cono Sur. La subregión Caribe no mantuvo una asociación significativa.

También se encontró que, con 1\% de significancia, aumentar el gasto militar como porcentaje del PIB de $2,06 \%$ a $3,06 \%$ se correlaciona con una probabilidad de $7 \%$ de disputas militarizadas. En este Modelo los límites fronterizos no ofrecen una asociación lineal con la probabilidad de disputas anuales por país. Finalmente, con $1 \%$ de significancia, ser un Estado libre se asocia con una probabilidad de $-69,83 \%$ disputas militarizadas, y con 5\% de significancia, ser un Estado parcialmente libre se asocia con una probabilidad de $-54,7 \%$ disputas anuales por país en comparación con los Estado no libres. La función distribución de los Estados en subregiones fue de 13,8\% en América del Norte; 22,7\% en América Central; $22,3 \%$ en la Zona Andina; $13.6 \%$ en el Caribe y $27 \%$ en el resto (Cono Sur, Surinam y Guyana francesa).

\section{Discusión de los resultados iCRS o CPS?}

El análisis de las disputas interestatales militarizadas y de los documentos programáticos de la defensa como indicadores de las prácticas de seguridad hacen evidente que la hipótesis de conflicto interestatal ha continuado presente en el continente (Thies, 2015). A su vez, permite reconocer que las prácticas de seguridad se diferencian en el nivel subregional generando mayores retos en términos de los procesos de integración en materia de seguridad y defensa, concretamente en la zona Andina y América Central.

Las estadísticas descriptivas señalan que las disputas militarizadas se presentaron anualmente entre 1992 y 2010, con una disminución de su incidencia durante la primera década del siglo XXI respecto de la predecesora. Estas disputas se concentraron en las subregiones donde interactúan los principales líderes regionales, no así en sus zonas de influencia directa: las disputas militarizadas ocurridas tanto en América del Norte como en el Cono Sur fueron escasas y dirigidas fundamentalmente hacia actores extra continentales. No obstante las subregiones de América Central y la zona Andina evidenciaron niveles importantes de hostilidad que se vinculan con los problemas de seguridad propios de su entorno: conflictos limítrofes irresueltos, incursiones militares en territorio soberano de otros Estados y crisis propiciadas por la operación de actores ilegales transnacionales. La amenaza o el uso de la violencia sumado a la persistencia en las normas domésticas de la amenaza de la agresión externa ha sido incluso justificación para la 
permanencia de la institución castrense en algunos Estados (Dassel, 1998).

Los modelos econométricos permiten identificar la correlación y la probabilidad de las disputas militarizadas mediante variables independientes derivadas de la literatura académica. El Modelo 1 destacó que, en orden descendiente, América del Norte, la zona Andina y América Central son más propensos a este tipo de prácticas de seguridad en comparación con el Cono Sur. El escenario de América del Norte se explica por la proyección estadounidense a escala global, pues este indicador no discierne las disputas dentro del continente de aquellas extra continentales. Asimismo, se da cuenta de la persistencia de las hipótesis realistas por cuanto el gasto militar como porcentaje del PIB y la mayor o menor cantidad de fronteras se asocian linealmente con un aumento o disminución de disputas, lo cual se explica por la mayor o menor posibilidad de fricciones interestatales. En oposición a lo argumentado por Domínguez, la democracia liberal sí genera menores posibilidades de disputas militarizadas al compararse con los Estados no libres. Por otra parte, el Modelo 2 corrobora la probabilidad de disputas militarizadas por subregiones, manteniendo la jerarquía de América del Norte (56\%), zona Andina (38\%) y América Central (26\%) en comparación con el Cono Sur, se corrobora la probabilidad de aumentar las disputas militarizadas en
$7 \%$ ante un aumento de $1 \%$ de gasto militar como porcentaje del PIB, y se corrobora la hipótesis liberal de que la mayor libertad (democracia liberal) reduce la probabilidad de disputas frente a los Estados que no son libres. No obstante, no respalda al argumento de que la cantidad de fronteras aumenta la probabilidad de disputas.

Estos hallazgos descritos se enmarcan en la discusión sobre los tipos de paces prevalecientes en la región como causa subyacente de los sistemas de seguridad. En estos términos Mares ha considerado que "no ha habido una larga paz en la región, si se define la paz como la ausencia de 'guerra' o la ausencia de graves enfrentamientos militares" (2001, p. 4), pero Cairo y Lois (2014, p. 47) argumentan que "presentar en términos muy alarmantes estas disputas" no es aceptable, pues si bien "algunas han producido escaladas de tensión... también es verdad que otras se han solucionado pacíficamente", y PionBerlin y Trinkunas señalaron que las disputas militarizadas son pequeñas en escala y no han desatado un interés civil significativo en términos de la política de defensa (2007, p. 85).

Asimismo, diversos autores han propuesto conceptos para distinguir la región frente a la prevalencia de conflictos y la presencia de instituciones sólidas que permiten su resolución. En este sentido Battaglino propone la paz híbrida pues considera insatisfactorio hablar solamente de paz 
negativa o positiva por la presencia o ausencia de conflicto violento (2012, p. 133), ya que desmerece el papel de la democracia, el crecimiento económico y las instituciones. Por otra parte Mares utiliza el concepto de "paz violenta" (2001), que busca resaltar la ausencia de una gran guerra a pesar de los frecuentes enfrentamientos violentos, o bien el trabajo de Martín (2006) habla de una "paz militarista" señalando que una gran guerra no ha ocurrido debido a que los sectores militares no han tenido motivaciones para buscar beneficios mediante el uso externo de la fuerza (2006, pp. 5-6).

A raíz de estas discusiones vigentes se considera que, al estudiar el continente resulta más apropiado su abordaje a partir de los Complejos regionales de seguridad dada la persistencia de la amenaza y uso de la fuerza como prácticas de seguridad. No obstante, como se ha destacado mediante la evidencia empírica los análisis subregionales generan perspectivas distintas respecto del conjunto, de tal forma que América Central y la zona Andina pueden comprenderse como "subcomplejos" y zonas de interacción de los principales líderes regionales. En estas subregiones el concepto de paz híbrida permite mejorar la precisión analítica pues suceden disputas militarizadas pero también se desarrolla progresivamente la democracia, se sostiene el intercambio económico y la participación en las instituciones internacionales.
Si se reflexiona respecto de América del Norte se encuentran trabajos que, a pesar de reconocer en el nivel transnacional la posibilidad de estarse constituyendo una CPS, la primacía de la agenda de seguridad estadounidense determina que no se podría entender su dinámica securitaria sino es mediante el CRS (Frasson-Quenoz, 2014), y cómo la proyección de la fuerza de Estados Unidos en el nivel internacional continua genera amenazas especialmente transnacionales para los Estados adyacentes. Por su parte el Cono Sur representa la región más estable y es por ello que la Comunidad pluralista de seguridad sí resulta un concepto útil para explicar la ausencia de disputas militarizadas y la creciente interdependencia entre sus Estados (Oelsner, 2015, p. 182) enmarcada en la posguerra fría y el advenimiento de la tercera ola de la democratización en la subregión (Ovando Santana, 2012).

\section{Conclusiones}

Los estudios sobre el nuevo regionalismo de seguridad en el continente americano durante la posguerra fría han fallado en aportar evidencia empírica comparada para determinar si se presencian CRS o CPS. Frente a este dilema analítico el estudio ha optado ontológicamente por dar privilegio a la dimensión fácticainductiva estudiando las prácticas de seguridad y no lo deontológico que emana de las aspiraciones políticas. 
Para ello se ha destacado cómo las aproximaciones regionalistas de seguridad se han diferenciado frente al nuevo regionalismo por cuanto continúan dando relevancia a la dimensión material (del poder, la geografía y del uso de la violencia).

Si bien es cierto los CRS y las CPS se diferencia meta-teóricamente por sus tipos de culturas anárquicas, tipos de paz y el balance que ofrecen entre lo material y lo ideacional, se ha considerado que la principal diferencia en América puede discernirse en lo fáctico, pues los Estados han sido socializados en la misma arquitectura de seguridad siendo ello una constante, mientras que la amenaza y el uso de la fuerza armada presenta variaciones regionales y subregionales.

Para aprehender las prácticas de seguridad se utilizaron dos indicadores: las disputas interestatales militarizadas y complementariamente los instrumentos programáticos de la defensa nacional identificando la persistencia de la hipótesis de conflicto interestatal.

La evidencia cuantitativa permite concluir que, al estudiar el continente resulta más apropiado el abordaje a partir de los CRS dada la persistencia de la amenaza y uso de la fuerza como prácticas de seguridad.

No obstante, esta misma evidencia empírica destaca que los análisis subregionales generan perspectivas distintas respecto del conjunto, de tal forma que América Central y la zona Andina pueden comprenderse como subcomplejos y zonas de interacción de los principales líderes regionales, mientras que en América del Norte la primacía de la agenda de seguridad estadounidense en su subregión inmediata y mediata (América Central y Caribe) implican al CRS, especialmente por su proyección de poder e influencia mundial.

Por su parte el Cono Sur representa la región más estable y es por ello que la Comunidad pluralista de seguridad resulta un concepto útil para explicar la ausencia de disputas militarizadas y la creciente interdependencia entre sus Estados, no obstante al asociarse con los Estados de la zona Andina constituye un CRS por la adición de los problemas de seguridad propios de esta subregión.

\section{Referencias}

Ayoob, M. (2005). Security in the Age of Globalization: Separating Appearance from Reality. En E. Aydinli \& J. N. Rosenau (Eds.), Globalization, Security, and the Nation State: Paradigms in Transition (pp. 9-26). Albany: State University of New York Press.

Battaglino, J. M. (2012). The coexistence of peace and conflict in South America: toward a new 
conceptualization of types of peace. Revista Brasileira de Política Internacional, 55 (2), 131-151. http://doi.org/10.1590/S003473292012000200008

Bonilla, A. (Ed.). (1999). Ecuador-Perú. Horizontes de la negociación y el conflicto. Quito: FLACSO Ecuador.

Breslin, S., Higgott, R., \& Rosamond, B. (2003). Regions in comparative perspective. En S. Breslin, C. W. Hughes, N. Phillips, \& B. Rosamond (Eds.), New Regionalism in the Global Political Economy: Theories and Cases (pp. 1-19). Londres: Routledge.

Buzan, B., \& Wæver, O. (2003). Regions and Powers: The Structure of International Security. Cambridge: Cambridge University Press.

Cairo, H., \& Lois, M. (2014). Geografía política de las disputas de fronteras: cambios y continuidades en los discursos geopolíticos en América Latina (1990-2013). Cuadernos de Geografía: Revista Colombiana de Geografía, 23 (2), 45-67. http://doi. org/10.15446/rcdg.v23n2.39578

Collier, P. (1999). Economic Causes of Civil War and Their Implications for Policy. Banco Mundial. Recuperado a partir de http://web.worldbank. org/archive/website01241/WEB/ IMAGES/ECONONMI.PDF

Dassel, K. (1998). Civilians, Soldiers, and Strife: Domestic Sources of International Aggression. International Security, 23 (1), 107--140.
Domínguez, J. I. (Ed.). (1998). Seguridad internacional, paz y democracia en el Cono Sur. Santiago: FLACSO Chile.

Fawcett, L. L. (2005). Regionalism from a Historical Perspective. En M. Farrell, B. Hettne, \& L. van Langenhove (Eds.), Global politics of regionalism: theory and practice (pp. 21-38). Londres: Pluto Press.

Frasson-Quenoz, F. (2013). Funcionamiento de los Complejos Regionales Estatales: un comparativo África/América del Sur y el Caribe. Contra Relatos desde El Sur. Apuntes Sobre África Y Medio Oriente, 11, 55-75.

Frasson-Quenoz, F. (Ed.). (2014). Seguridad internacional $y$ ordenamientos regionales: del Complejo Regional a la Comunidad de Seguridad. Europa, África, América del Sur y América del Norte. Bogotá: Universidad Externado de Colombia.

Gibler, D. M., \& Tir, J. (2010). Settled Borders and Regime Type: Democratic Transitions as Consequences of Peaceful Territorial Transfers. American Journal of Political Science, 54 (4), 951-968. http://doi.org/10.1111/ j.1540-5907.2010.00473.x

Gómez-Mera, L. (2008). How "new" is the "New Regionalism" in the Americas? The case of MERCOSUR. Journal of International Relations and Development, 11 (3), 279-308. http://doi.org/10.1057/jird.2008.14 
Gortzak, Y., Haftel, Y. Z., \& Sweeney, K. (2005). Offense-Defense Theory An Empirical Assessment. Journal of Conflict Resolution, 49 (1), 67-89. http://doi. org/10.1177/0022002704271280

Hensel, P. (1998). Reliability and Validity Issues in the ICOW Project. En Annual Meeting of the International Studies Association. Minneapolis.

Herz, M. (2008). Does the Organisation of American States Matter? (Crisis States Working Papers Series No. 2). Londres: Crisis States Research Centre.

Hettne, B. (2004). The New Regionalism Revisited. En F. Söderbaum \& T. M. Shaw (Eds.), Theories of New Regionalism: A Palgrave Reader (pp. 22-42). Londres: Palgrave Macmillan.

Hettne, B., \& Söderbaum, F. (2003). Regions in comparative perspective. En S. Breslin, C. W. Hughes, N. Phillips, \& B. Rosamond (Eds.), New Regionalism in the Global Political Economy: Theories and Cases (pp. 33-47). Londres: Routledge.

IDEA Internacional. (2014). La calidad de las democracias en América Latina. San José: IDEA Internacional. Recuperado a partir de http://www.idea.int/es/ publications/sod-in-latin-america/ index.cfm?css= new2013

Jones, D. M., Bremer, S. A., \& Singer, J. D. (1996). Militarized Interstate
Disputes, 1816-1992: Rationale, Coding Rules, and Empirical Patterns. Conflict Management and Peace Science, 15 (2), 163213. http://doi.org/10.1177/07388 9429601500203

Kelly, R. E. (2007). Security Theory in the "New Regionalism". International Studies Review, 9 (2), 197-229. http://doi.org/10.1111/ j.1468-2486.2007.00671.x

Li, Q., \& Sacko, D. H. (2002). The (Ir) Relevance of Militarized Interstate Disputes for International Trade. International Studies Quarterly, 46 (1), 11-43. http://doi. org/10.1111/1468-2478.00221

Mares, D. R. (2001). Violent Peace: Militarized Interstate Bargaining in Latin America. Nueva York: Columbia University Press.

Mares, D. R. (2003). Conflictos limítrofes en el Hemisferio Occidental: Análisis de su relación con la estabilidad democrática, la integración económica y el bienestar social. En J. I. Domínguez (Ed.), Conflictos territoriales y democracia en América Latina (pp. 47-84). Buenos Aires: Siglo Veintiuno Editores Argentina.

Mares, D. R. (2014). Interstate Security Issues in Latin America. En J. I. Dominguez \& A. Covarrubias (Eds.), Routledge Handbook of Latin America in the World (pp. 420-433). Nueva York: Routledge.

Mares, D. R., \& Bernstein, S. (1998). El 
uso de la fuerza en las relaciones interestatales de América Latina. En J. I. Domínguez (Ed.), Seguridad internacional, paz y democracia en el Cono Sur (pp. 49-80). Santiago: FLACSO Chile.

Martín, F. E. (2006). Militarist Peace in South America: Conditions for War and Peace. Nueva York: Palgrave Macmillan.

Méndez-Coto, M. V. (2012). ¿Poder inteligente? La doctrina Obama y Guantánamo. Heredia: Universidad Nacional de Costa Rica.

Méndez-Coto, M. V., \& Cortez, C. (2014). Gobernanza subregional de las migraciones y relaciones internacionales: los casos del CA4 y el MERCOSUR. En W. Soto Acosta (Ed.), Política Internacional e Integración Regional Comparada en América Latina (pp. 213-231). San José: FLACSO Costa Rica.

Méndez-Coto, M. V. (2015). Poder suave, poder duro y poder inteligente. Una aproximación desde Costa Rica. En W. Soto Acosta (Ed.), Ciencias Sociales y Relaciones Internacionales: nuevos enfoques desde América Latina (pp. 303-330). Heredia: Universidad Nacional de Costa Rica.

Méndez-Coto, M. V. (2016a). Multilateralismo, gobernanza y hegemonía en la estructura económica internacional: del G7 al G20. Revista Académica de Relaciones Internacionales, (31), 13-32.
Méndez-Coto, M. V. (2016b). Reseña a Seguridad internacional y ordenamientos regionales: del Complejo Regional a la Comunidad de Seguridad. Temas de Nuestra América. Revista de Estudios Latinoamericanos, 32 (59), 155-158.

Oelsner, A. (2015). Pluralistic security communities in Latin America. En A. M. Kacowicz \& D. R. Mares (Eds.), Routledge Handbook of Latin American Security (pp. 173184). Nueva York: Routledge.

Ovando Santana, C. (2012). La seguridad internacional en la proyección de Chile hacia el Cono Sur: ¿desde la doctrina de la seguridad nacional hacia la construcción de Comunidades de Seguridad o la emergencia de la securitización?. Revista de Relaciones Internacionales, Estrategia y Seguridad, 7 (2), 193-219. doi:http://dx.doi. org/10.18359/ries.88

Paul, T. V. (1994). Asymmetric Conflicts: War Initiation by Weaker Powers. Nueva York: Cambridge University Press.

Pion-Berlin, D., \& Trinkunas, H. A. (2007). Attention Deficits: Why Politicians Ignore Defense Policy in Latin America. Latin American Research Review, 42 (3), 76100. http://doi.org/10.1353/ lar.2007.0031

Ramsbotham, O., Miall, H., \& Woodhouse, T. (2011). 
Contemporary Conflict Resolution: The Prevention, Managment and Transformation of Deadly Conflicts (Tercera edición). Cambridge: Polity.

RESDAL. (2014). Atlas Comparativo de la Defensa en América Latina y Caribe. Buenos Aires: RESDAL. Recuperado a partir de http:// www.resdal.org/assets/atlas-2014completo.pdf

Simmons, B. A. (2006). Trade and Territorial Conflict in Latin America: International Borders as Institutions. En M. Kahler \& B. Walter (Eds.), Territoriality and
Conflict in an Era of Globalization (pp. 251-287). Cambridge: Cambridge University Press.

Thies, C. G. (2015). Traditional security: war and peace. En A. M. Kacowicz \& D. R. Mares (Eds.), Routledge Handbook of Latin American Security (pp. 113-126). Nueva York: Routledge.

Wallensteen, P. (2011). Understanding Conflict Resolution: War, Peace and the Global System (Tercera edición). Londres: SAGE.

Wendt, A. (1999). Social Theory of International Politics. Nueva York: Cambridge University Press. 